\title{
IncRNA IUR upregulates miR-34a to inhibit pancreatic adenocarcinoma cell migratory and invasive abilities
}

\author{
REN LI ${ }^{1}$, JIAN ZHANG ${ }^{1}$, SHIYANG MA ${ }^{2}$, GANG ZHAO $^{2}, \mathrm{JUN} \mathrm{LI}^{3}$, \\ JIANGWEI LI $^{3}$, XIAOYI WANG ${ }^{3}$ and BO HUI ${ }^{1}$ \\ Departments of ${ }^{1}$ Hepatobiliary Surgery and ${ }^{2}$ Gastroenterology; \\ ${ }^{3}$ National and Local Joint Engineering Research Center of Biodiagnostics and Biotherapy, \\ The Second Affiliated Hospital of Xi'an Jiaotong University, Xi'an, Shaanxi 710004, P.R. China
}

Received August 16, 2020; Accepted February 12, 2021

DOI: $10.3892 / \mathrm{ol} .2021 .12828$

\begin{abstract}
The long non-coding RNA (lncRNA) imatinib-upregulated (IUR) has been recently reported as a tumor suppressor in leukemia. Preliminary microarray data revealed a downregulation of IUR in pancreatic adenocarcinoma (PAAD) and a positive correlation with microRNA-34a (miR-34a) expression. The present study aimed to investigate the role of IUR in PAAD. This study included samples from 58 patients with PAAD and the PAAD cell lines Capan-2 and HPAC. Reverse transcription quantitative PCR was performed to determine gene expression levels. Cell transfections were carried out to assess gene interactions between IUR, miR-34a and CD44. Transwell assays were performed to explore the effects of transfections on cell invasive and migratory abilities. The results demonstrated that IUR was downregulated in PAAD tissue compared with adjacent non-tumor tissue samples and that low expression levels of IUR correlated with poor survival in patients with PAAD. In PAAD tissue samples, the expression of IUR positively correlated with miR-34a expression but negatively correlated with CD44 expression, which is a target of miR-34a. In PAAD cells, overexpression of IUR resulted in miR-34a upregulation and CD44 downregulation. miR-34a overexpression did not affect the expression of IUR but downregulated CD44. In PAAD cells, overexpression of IUR and miR-34a led to decreased invasive and migratory abilities. However, CD44 overexpression played an opposite role and attenuated the effects of IUR and miR-34a overexpression. In conclusion, the results from this study demonstrated that IUR may upregulate miR-34a expression in order to inhibit PAAD cell migration and invasion by downregulating CD44.
\end{abstract}

Correspondence to: Dr Jian Zhang, Department of Hepatobiliary Surgery, The Second Affiliated Hospital of Xi'an Jiaotong University, 157 West 5th Road, Xi'an, Shaanxi 710004, P.R. China

E-mail: zhangjian_xjtu@126.com

Key words: long non-coding RNA, imatinib-upregulated, pancreatic adenocarcinoma, microRNA-34a, CD44

\section{Introduction}

Most pancreatic cancer-associated deaths are attributable to pancreatic adenocarcinoma (PAAD) globally (1). PAAD is a common cause of cancer-associated mortality and only $<4 \%$ of patients can live longer than 5 years following diagnosis $(2,3)$. Furthermore, the incidence of PAAD is continuously increasing, and it is estimated that PAAD will become the second leading cause of cancer-associated mortality in the United States by 2030 (4). Pancreaticoduodenectomy is currently the only potentially curative therapeutic approach for PAAD $(5,6)$. Most types of cancer can usually be cured at local stages by surgical resection $(5,6)$. However, resections of PAAD tumor usually fail to achieve long-term survival in most cases $(5,6)$. It is therefore crucial to develop novel therapeutic approaches for PAAD.

Genetic factors play pivotal roles in the development and progression of PAAD (7). As a cell-surface glycoprotein, CD44 serves a crucial role in cell-cell interactions (8). CD44 is a multifunctional cellular factor that participates in diverse biological processes, such as recirculation, hematopoiesis, lymphocyte activation and tumor metastasis (9). In PAAD, CD44 interacts with multiple oncogenic or tumor suppressive regulators to promote cancer progression (10). In addition, certain tumor suppressive microRNAs (miRNAs), including miR-34a, targets CD44 to suppress cancer metastasis (11). It has been reported that miRNAs can interact with other non-coding RNA molecules, such as long non-coding RNA (lncRNA; >200 nucleotides in length), to participate in cancer biology (12). Imatinib-upregulated lncRNA (IUR) is a novel lncRNA that has been reported as a tumor suppressor in leukemia (13). Preliminary microarray data from our laboratory revealed the downregulation of IUR in PAAD tissues and its positive correlation with miR-34a (data not shown). IUR may therefore have crosstalk with both miR-34a and CD44 in PAAD. Thus, the present study aimed to investigate the interactions among IUR, miR-34a and CD44 in PAAD, and to determine their effects on PAAD cell invasion and migration.

\section{Materials and methods}

Patient information. The present study included 58 patients with PAAD (28 men and 30 women; age range, 23-66 years; 
mean age, $45 \pm 16.1$ years) selected from 122 patients with PAAD who were admitted at The Second Affiliated Hospital of Xi'an Jiaotong University between January 2012 and January 2014. All patients were followed up from the date of admission until January 2019 to record their survival conditions. Follow-up was performed every month through outpatient visit and/or telephone calls. All PAAD cases were confirmed by histopathological analysis. The inclusion criteria were as follows: i) Patients newly diagnosed with PAAD; ii) patients with no other clinical disorders; and iii) patients who received no treatment for any disease six months prior to admission. The exclusion criteria were as follows: i) Patients with recurrent PAAD; ii) patients for whom therapies for PAAD were initiated; ii) patients with other severe clinical disorders, and iii) stage IV patients. According to the American Joint Committee on Cancer staging systems (14), there were 20, 23 and 15 cases at stage I, II and III, respectively. This study was approved by The Ethics Committee of The Second Affiliated Hospital of Xi'an Jiaotong University. All patients were informed with the details of this study and signed written informed consent.

PAAD tissues and cells. The human PAAD cell lines Capan-2 and HPAC (American Type Culture Collection) were used in this study. Cells were cultured in McCoy's 5a Medium (Thermo Fisher Scientific, Inc.) containing 10\% FBS (Thermo Fisher Scientific, Inc.) and placed at $37^{\circ} \mathrm{C}$ in a humidified incubator containing $5 \% \mathrm{CO}_{2}$. Biopsy was performed on all 58 patients with PAAD to collect PAAD lesion tissue and adjacent non-cancer tissue samples within $3 \mathrm{~cm}$ around tumors. All tissue samples were stored in liquid nitrogen before use. Histopathological examination was performed to test all tissue specimens. Non-cancer tissues contained $<1 \%$ cancer cells and PAAD tissues contained $>95 \%$ cancer cells.

Transient transfection. The pcDNA3.1 vector was used as backbone to construct IUR and CD44 expression vectors by Guangzhou RiboBio Co., Ltd. Negative control (NC) miRNA (5'-UGCGACGUUGGACGUGACGAAU-3') and miR-34a mimic (5'-UGGCAGUGUCUUAGCUGGUUGU-3') were synthesized by Sangon Biotech Co., Ltd. Capan-2 and HPAC cells were harvested at $70-80 \%$ confluence and $5 \times 10^{5}$ cells were transfected with $10 \mathrm{nM}$ plasmids (pcDNA3.1-IUR, pcDNA3.1-CD44 vectors or empty pcDNA3.1 vector as NC) or $30 \mathrm{nM}$ miR-34a mimic (or negative control miRNA as NC). All transfections were performed through transient transfections using Lipofectamine ${ }^{\circledR} 2000$ (Sangon Biotech Co., Ltd.) for $6 \mathrm{~h}$ at $37^{\circ} \mathrm{C}$. Cells were collected at $24 \mathrm{~h}$ post-transfection for subsequent experiments. Untransfected cells were used as the control (C) group.

Total RNA and miRNA extractions. The weight of tissues ranged from 0.016 to $0.021 \mathrm{~g}$. Tissue specimens $(0.015 \mathrm{~g})$ were ground in liquid nitrogen. Capan- 2 cells were harvested and counted, and $1 \times 10^{6}$ cells were collected. Total RNA and miRNA were extracted from tissues and cells using Trizol ${ }^{\circledR}$ (Invitrogen; Thermo Fisher Scientific, Inc.) according to the manufacturers' instructions. During the precipitation and washing step, $85 \%$ ethanol was used to precipitate miRNA.
Reverse transcription quantitative $(R T-q) P C R$. RNA samples were digested with DNase I to remove genomic DNA. Tetro Reverse Transcriptase (Bioline) was used to perform reverse transcriptions ( $1 \mu \mathrm{g}$ RNA per reaction, $25^{\circ} \mathrm{C}$ for $10 \mathrm{~min}, 52^{\circ} \mathrm{C}$ for $30 \mathrm{~min}$ and $85^{\circ} \mathrm{C}$ for $10 \mathrm{~min}$ ). qPCR reaction mixtures were prepared using BlazeTaq ${ }^{\mathrm{TM}}$ SYBR-Green qPCR Mix (GeneCopoeia, Inc.). GAPDH was used as the endogenous control to measure the expression levels of IUR and CD44.

For miRNA qPCR, MystiCq ${ }^{\circledR}$ microRNA cDNA Synthesis Mix (Sigma-Aldrich; Merck KGaA) was used to perform all reverse transcription. The qPCR reaction mixtures were prepared using miScript SYBR Green PCR Kit (Qiagen China Co., Ltd). U6 was used as the endogenous control to measure the expression level of miR-34a. All qPCR reactions were performed three times.

The primer sequences were as follows: IUR, forward 5'-AGCGGTTTCCTCTTGTTTGTG-3', reverse 5'-CTTTTG GGTGAGAAAACAAGCC-3'; CD44, forward 5'-ACCTGC CCAATGCCTTTGATGGA-3', reverse 5'-CAAAGCCAA GGCCAAGAGGGATG-3'; GAPDH, forward 5'-GCTTTC TTTCCTTTCGCGCT-3', reverse 5'-TTTGCGGTGGAAATG TCCTT-3'; and miR-34a, forward 5'-TGGCAGTGTCTTAGC TGGT-3'. Universal miRNA reverse primer and U6 forward primer were provided in the miScript SYBR-Green PCR Kit. PCR reactions were performed as follows: $95^{\circ} \mathrm{C}$ for $1 \mathrm{~min}$, and then $95^{\circ} \mathrm{C}$ for $10 \mathrm{sec}$ and $61^{\circ} \mathrm{C}$ for $40 \mathrm{sec}$.

The relative expression levels were normalized to endogenous control and were expressed as $2^{-\Delta \Delta \mathrm{Cq}}(15)$.

Western blotting. Capan-2 cells were harvested and counted. Cells $\left(1 \times 10^{6}\right)$ were lysed using RIPA solution on ice (Sangon Biotech Co., Ltd.). All protein samples were denatured in boiled water for $5 \mathrm{~min}$. Proteins $(20 \mu \mathrm{g})$ were separated by $12 \%$ SDS-PAGE and transferred onto PVDF membranes. Membranes were blocked using 5\% skimmed milked in PBS at $23^{\circ} \mathrm{C}$ for $1 \mathrm{~h}$ and were incubated with primary antibodies against $\mathrm{CD} 44$ (1:1,000; cat. no. ab157107; Abcam) and GAPDH (1:1,000; cat. no. ab37168; Abcam) at $4^{\circ} \mathrm{C}$ overnight. Membranes were then incubated with the goat anti-rabbit immunoglobulin G-HRP secondary antibody (1:1,000; cat. no. ab6721; Abcam) at $23^{\circ} \mathrm{C}$ for $2 \mathrm{~h}$. Signals were developed using RapidStep ${ }^{\mathrm{TM}}$ ECL detection reagent (Sigma-Aldrich; Merck KGaA). The data were analyzed via densitometry using ImageJ v1.48 software (National Institutes of Health) and normalized to expression of the internal control GAPDH.

Cell migration and invasion assays. Capan-2 and HPAC cells were harvested and counted. Cells $\left(3 \times 10^{4}\right)$ were mixed with $1 \mathrm{ml}$ McCoy's 5a Medium containing 1\% FBS to prepare cell suspensions. The Transwell upper chamber was filled with $0.1 \mathrm{ml}$ serum-free cell suspension, and the Transwell lower chamber was filled with McCoy's 5a Medium supplemented with 20\% FBS. Prior to invasion assay, membranes were coated with Corning ${ }^{\circledR}$ Matrigel ${ }^{\circledR}$ matrix (Corning) at $37^{\circ} \mathrm{C}$ for $6 \mathrm{~h}$. Transwell chambers were incubated at $37^{\circ} \mathrm{C}$ for $12 \mathrm{~h}$. Subsequently, the membranes were washed with PBS and cells were stained with $1 \%$ crystal violet (Sigma-Aldrich; Merck KGaA) at room temperature for $10 \mathrm{~min}$. The stained cells were counted under a light microscope. 

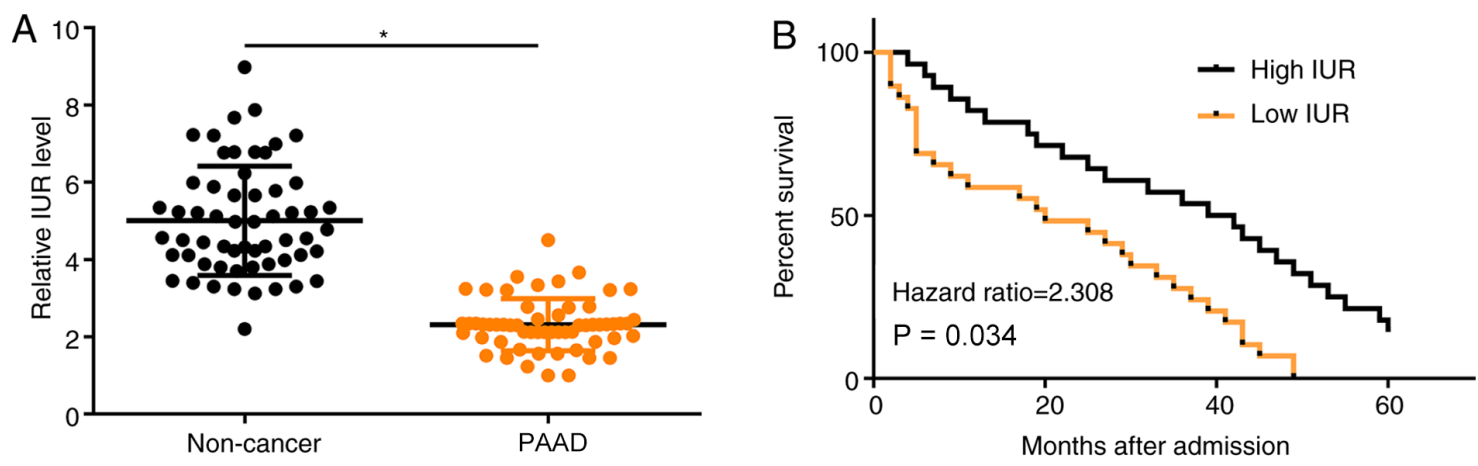

Figure 1. IUR is downregulated in PAAD and low expression of IUR is associated with the poor survival of patients with PAAD. $\mathrm{n}=3$. The data are presented as the mean \pm standard deviation. ${ }^{*} \mathrm{P}<0.05$. (A) Expression of IUR in PAAD and adjacent non-tumor tissues. (B) Survival curves for patients with high and low IUR expression. Patients in low IUR group had significantly lower overall survival rate compared with patients in high IUR group. IUR, imatinib-upregulated; PAAD, pancreatic adenocarcinoma.

Statistical analysis. GraphPad Prism 6 (GraphPad Software, Inc.) was used for data analysis. Western blotting, RT-qPCR, cell invasion and cell migration assays were repeated three times. Data are presented as the mean \pm standard deviation. Differences between two groups were compared using paired t-tests. Comparisons between three groups or more were done using one-way ANOVA followed by Tukey's post hoc test. Correlation analysis was performed by linear regression. The 58 patients with PAAD were divided into high- and low-expression groups using the median expression level of IUR in PAAD tissues as cutoff value (cutoff value, 2.11). KM plotter (https://kmplot.com/analysis/) was used for survival analysis, and survival curves were compared using log-rank test. $\mathrm{P}<0.05$ was considered to indicate a statistically significant difference.

\section{Results}

IUR is downregulated in PAAD tissues and low expression of IUR is associated with the poor survival of patients with PAAD. The expression of IUR in PAAD and adjacent non-tumor tissues was assessed by RT-qPCR. The results demonstrated that IUR expression was significantly decreased in PAAD tissues compared with non-cancer tissues (Fig. 1A; $\mathrm{P}<0.05$ ). Survival curves were obtained patients with high and low IUR expression. It was observed that patients in the low-expression group had a significantly lower overall survival rate compared with patients with high IUR expression (Fig. 1B; P<0.05).

IUR expression positively correlates with miR-34a expression but negatively correlated with $\mathrm{CD} 44$ expression. The expression of miR-34a and CD44 in PAAD tissue samples was evaluated by RT-qPCR. Correlation analysis demonstrated that the expression of IUR was positively correlated with the expression of miR-34a (Fig. 2A), but negatively correlated with the expression of CD44 (Fig. 2B) in PAAD tissues.

IUR downregulates CD44 by upregulating miR-34a. The IUR expression vector, miR-34a mimic and CD44 expression vector were transfected into Capan- 2 cells. At $24 \mathrm{~h}$ post-transfection, the expression of IUR, miR-34a and CD44 was significantly upregulated compared with the $\mathrm{C}$ and $\mathrm{NC}$ groups (Fig. 3A;
$\mathrm{P}<0.05)$. Furthermore, overexpression of IUR resulted in miR-34a upregulation, while overexpression of miR-34a did not affect the expression of IUR (Fig. 3B). In addition, IUR overexpression or miR-34a led to downregulated expression of CD44 in PAAD cells (Fig. 3C; P $<0.05$ ). However, overexpression of CD44 did not affect the expression of IUR and miR-34a (Fig. 3D).

IUR inhibits Capan-2 and HPAC cell invasive and migratory abilities via miR-34a and CD44. Cell invasion and migration assay results demonstrated that, compared with the $\mathrm{NC}$ and $\mathrm{C}$ groups, overexpression of IUR and miR-34a led to decreased invasive (Fig. 4A) and migratory (Fig. 4B) abilities of Capan-2 cells $(\mathrm{P}<0.05)$. Overexpression of $\mathrm{CD} 44$ played the opposite role and attenuated the effect of IUR overexpression $(\mathrm{P}<0.05)$.

Another PAAD cell line, HPAC, was used to repeat the Transwell assays to further confirm the role of IUR in regulating PAAD cell invasion and migration. Similarly, invasion (Fig. S1A) and migration (Fig. S1B) of HPAC cells were inhibited after the overexpression of IUR and miR-34a $(\mathrm{P}<0.05)$. Overexpression of $\mathrm{CD} 44$ played the opposite role and attenuated the effects of IUR overexpression (Fig. S1; P<0.05).

\section{Discussion}

The present study investigated the associations among IUR, miR-34a and CD44 in PAAD. The results demonstrated that IUR may be considered as an oncogenic lncRNA in PAAD and could inhibit cancer cell invasion and migration by downregulating CD44 through the upregulation of miR-34a, which can directly target CD44 (11).

The function of IUR has only been characterized in leukemia (13), in which IUR can regulate the STAT5-CD71 pathway to inhibit tumorigenesis induced by BCR-ABL1 (13). Our preliminary microarray data revealed downregulation of IUR in PAAD. In the present study, IUR was downregulated in PAAD and overexpression of IUR resulted in decreased invasive and migratory abilities of PAAD cells. IUR may therefore be considered as a tumor suppressor lncRNA in PAAD.

The survival of patients with PAAD has been improved in the last decades, although only $<5 \%$ of patients live longer than 5 years $(16,17)$. In the present study, according to the 5 -year follow-up, only four patients $(6.9 \%)$ survived, which 

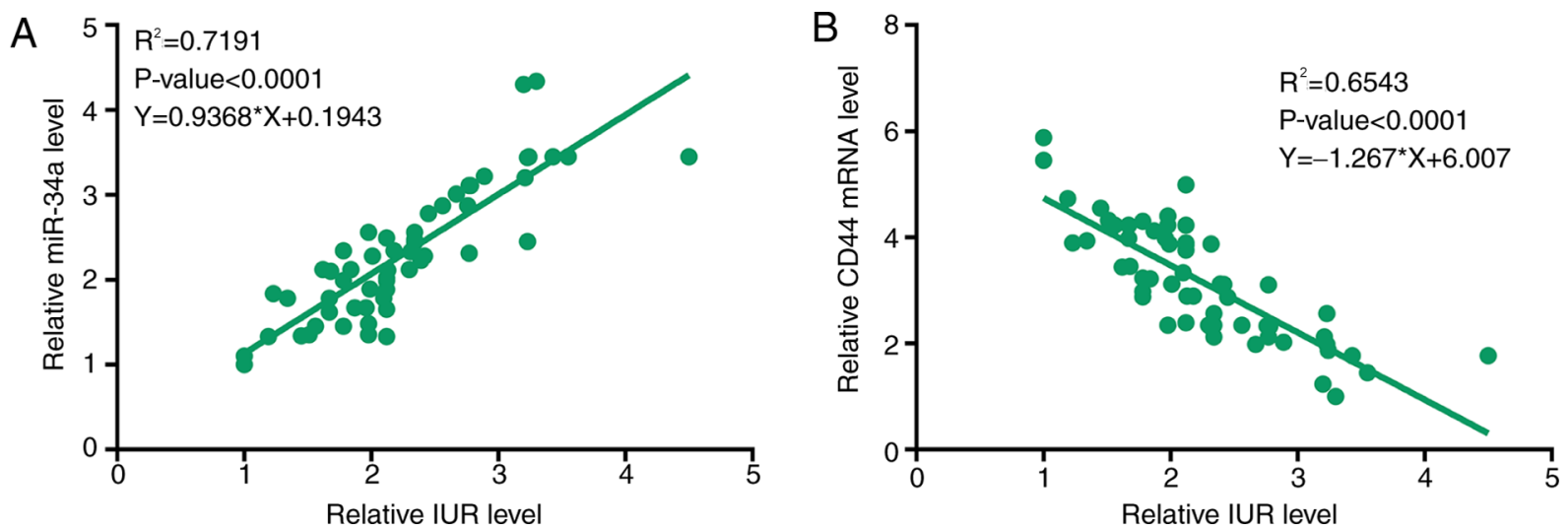

Figure 2. IUR positively correlates with miR-34a and negatively correlates with CD44. Expression of miR-34a and CD44 in PAAD tissues. Correlations between (A) IUR/miR-34a and (B) IUR/CD44 were analyzed using linear regression. miR, microRNA; IUR, imatinib-upregulated; PAAD, pancreatic adenocarcinoma.
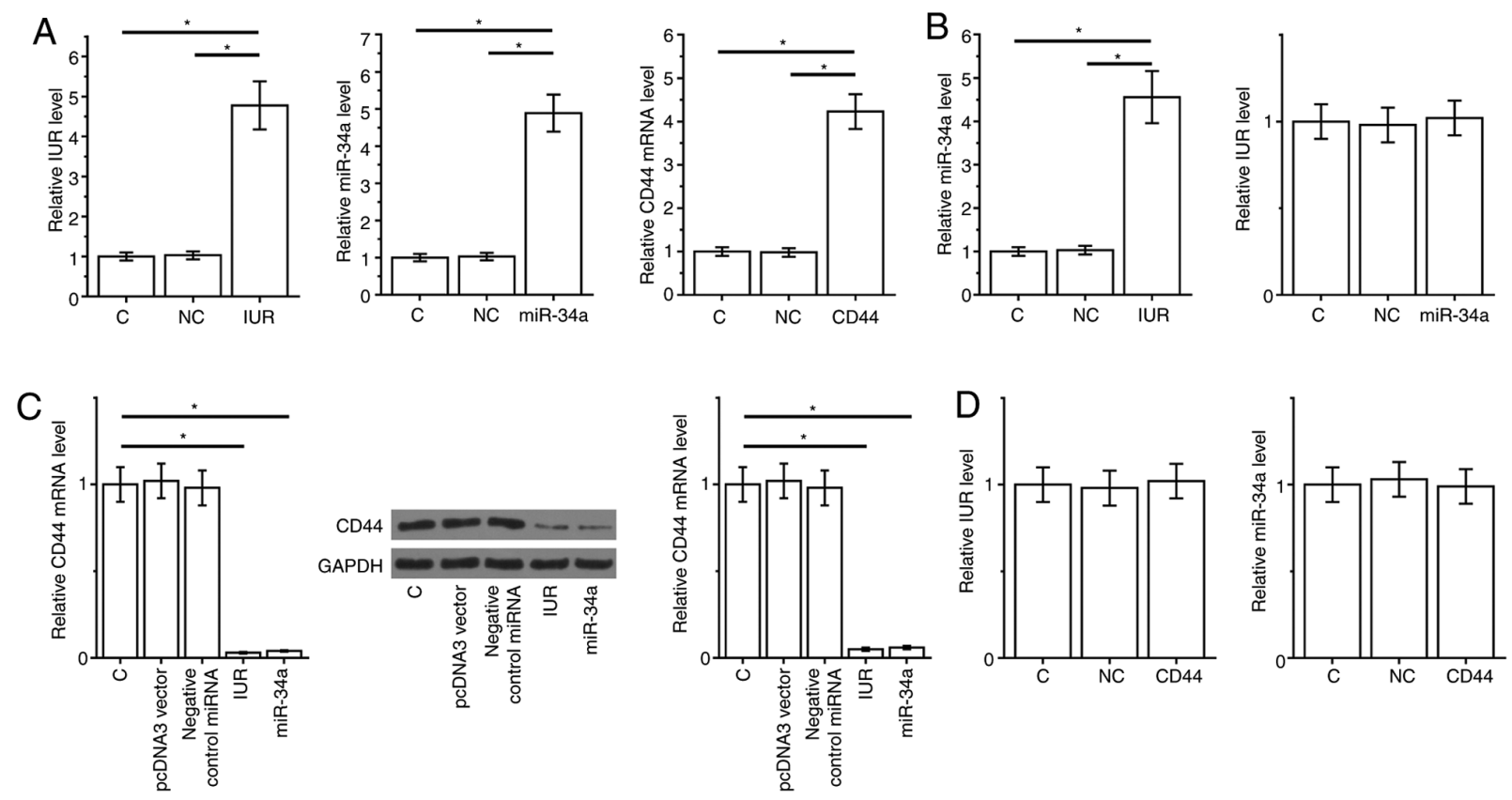

Figure 3. IUR may downregulate CD44 by upregulating miR-34a. IUR expression vector, miR-34a mimic and CD44 expression vector were transfected into Capan-2 cells. (A) At $24 \mathrm{~h}$ post-transfection, the expression of IUR, miR-34a and CD44 were measured. (B) IUR and miR-34a levels following transfection with miR-34a or IUR overexpression. (C) Protein and mRNA levels of CD44 following overexpression of IUR and miR-34a. (D) IUR and miR-34a levels following CD44 overexpression. " $\mathrm{P}<0.05 . \mathrm{n}=3$. The data are presented as the mean \pm standard deviation. $\mathrm{C}$, control; NC, negative control; IUR, imatinib-upregulated; miR, microRNA.

is slightly higher than the average $(4 \%)$ global level $(2,3)$. This could be due to the fact that this study only included patients at clinical stages I-III. Due to the extremely poor survival of these patients, accurate prognostic assignment is extremely important (18). The present study demonstrated that patients with low IUR expression in PAAD tissues experienced significantly lower overall survival rate. Evaluation of IUR expression may therefore assist the prognosis of patients with PAAD.

It has been reported that miR-34a can target CD44 in prostate cancer (11). In the present study, expression of CD44 was downregulated following miR-34a overexpression, both at the mRNA and protein levels. miR-34a may therefore also target CD44 in PAAD. In addition, this study reported that IUR could upregulate
miR-34a. However, no potential target of miR-34a on IUR was observed. Both IUR and miR-34a are known to have crosstalk with the STAT signaling pathway $(13,19)$. Therefore, the STAT signaling pathway may mediate the interaction between miR-34a and IUR. This will be further investigated in the future.

In conclusion, the present study demonstrated that IUR was upregulated in PAAD tissues compared with non-cancer tissue samples. In addition, IUR may upregulate miR-34a in PAAD cells to downregulate CD44, thereby inhibiting the invasive and migratory abilities of PAAD cancer cells.

\section{Acknowledgements}

Not applicable. 

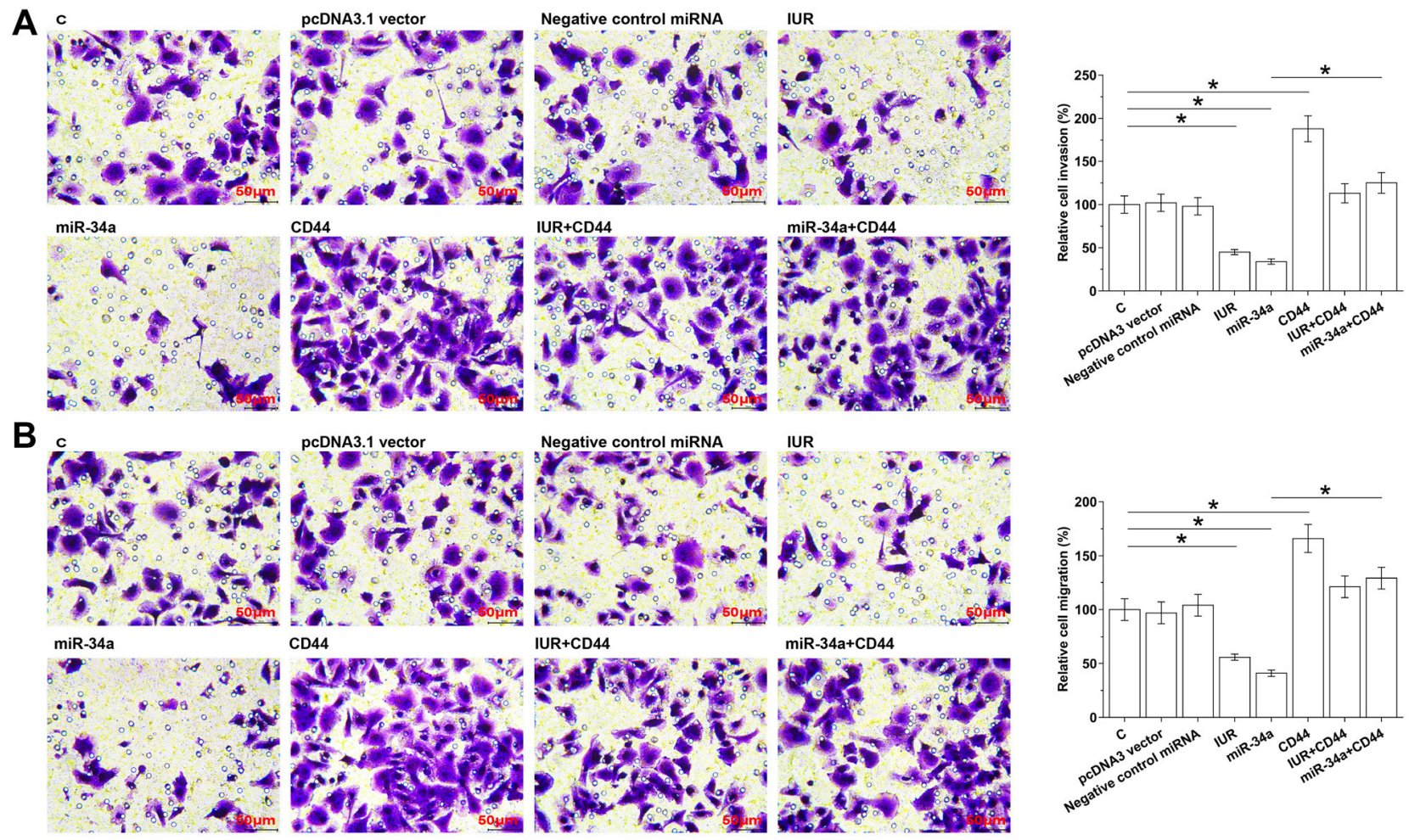

Figure 4. IUR inhibits Capan-2 cell invasive and migratory abilities through miR-34a and CD44. Roles of IUR, miR-34a and CD44 in regulating the (A) invasive and (B) migratory abilities of Capan-2 cells were examined using Transwell assays. Scale bar $=50 \mu \mathrm{m}$. " $\mathrm{P}<0.05$. $\mathrm{n}=3$. The data are presented as the mean \pm standard deviation. C, control; IUR, imatinib-upregulated; miR, microRNA.

\section{Funding}

The present study was supported by The Science and Technology Program of Shaanxi Province of China (grant no. 2014K11-03-03-07) and The Natural Science Foundation of Shaanxi Province of China (grant no. 2016JM8093). The funding bodies had no role in the design of the study; collection, analysis and interpretation of data and writing of the manuscript.

\section{Availability of data and materials}

All data generated or analyzed during this study are included in this published article.

\section{Authors' contributions}

$\mathrm{RL}$ and $\mathrm{SM}$ wrote the manuscript, performed literature research and analyzed data. GZ, JL, JWL, XW and $\mathrm{BH}$ analyzed data, performed the experiments and provided statistical analysis. JZ performed literature research and was responsible for project management and study design. RL, JZ, SM, GZ, JL JWL, XW and BH confirm the authenticity of the data in the present manuscript. All authors read and approved the final version.

\section{Ethics approval and consent to participate}

The Ethics Committee of the Second Affiliated Hospital of Xi'an Jiaotong University approved this study. All procedures performed in studies involving human participants were in accordance with the 1964 Declaration of Helsinki and its later amendments or comparable ethical standards. Written informed consent was obtained from all individual participants included in the study.

\section{Patient consent for publication}

Not applicable.

\section{Competing interests}

The authors declare that they have no competing interests.

\section{References}

1. Bray F, Ferlay J, Soerjomataram I, Siegel RL, Torre LA and Jemal A: Global cancer statistics 2018: GLOBOCAN estimates of incidence and mortality worldwide for 36 cancers in 185 countries. CA Cancer J Clin 68: 394-424, 2018.

2. Siegel RL, Miller KD and Jemal A: Cancer statistics, 2019. CA Cancer J Clin 69: 7-34, 2019

3. Luberice K, Downs D, Sadowitz B, Ross S and Rosemurgy A: Has survival improved following resection for pancreatic adenocarcinoma? Am J Surg 214: 341-346, 2017.

4. Rahib L, Smith BD, Aizenberg R, Rosenzweig AB, Fleshman JM and Matrisian LM: Projecting cancer incidence and deaths to 2030: The unexpected burden of thyroid, liver, and pancreas cancers in the United States. Cancer Res 74: 2913-2921, 2014.

5. Nickel F, Haney CM, Kowalewski KF, Probst P, Limen EF, Kalkum E, Diener MK, Strobel O, Müller-Stich BP and Hackert T: Laparoscopic versus open pancreaticoduodenectomy: A systematic review and meta-analysis of randomized controlled trials. Ann Surg 271: 54-66, 2020. 
6. Kamisawa T, Wood LD, Itoi T and Takaori K: Pancreatic cancer. Lancet 388: 73-85, 2016.

7. Amundadottir LT: Pancreatic cancer genetics. Int J Biol Sci 12: 314-325, 2016.

8. Aruffo A, Stamenkovic I, Melnick M, Underhill CB and Seed B: CD44 is the principal cell surface receptor for hyaluronate. Cell 61: 1303-1313, 1990.

9. Senbanjo LT and Chellaiah MA: CD44: A multifunctional cell surface adhesion receptor is a regulator of progression and metastasis of cancer cells. Front Cell Dev Biol 5: $18,2017$.

10. Jiang W, Zhang Y, Kane KT, Collins MA, Simeone DM, di Magliano MP and Nguyen KT: CD44 regulates pancreatic cancer invasion through MT1-MMP. Mol Cancer Res 13: 9-15, 2015.

11. Liu C, Kelnar K, Liu B, Chen X, Calhoun-Davis T, Li H, Patrawala L, Yan H, Jeter C, Honorio S, et al: The microRNA miR-34a inhibits prostate cancer stem cells and metastasis by directly repressing CD44. Nat Med 17: 211-215, 2011.

12. Jalali S, Bhartiya D, Lalwani MK, Sivasubbu S and Scaria V: Systematic transcriptome wide analysis of lncRNA-miRNA interactions. PLoS One 8: e53823, 2013.

13. Li H, Xu Y, Wang G, Chen X, Liang W and Ni H: Long non-coding RNA Mirt2 relieves lipopolysaccharide-induced injury in PC12 cells by suppressing miR-429. J Physiol Biochem 75: 403-413, 2019.
14. Appel BL, Tolat P, Evans DB and Tsai S: Current staging systems for pancreatic cancer. Cancer J 18: 539-549, 2012.

15. Livak KJ and Schmittgen TD: Analysis of relative gene expression data using real-time quantitative PCR and the 2(-Delta Delta C(T)) method. Methods 25: 402-408, 2001.

16. Von Hoff DD, Ervin T, Arena FP, Chiorean EG, Infante J, Moore M, Seay T, Tjulandin SA, Ma WW, Saleh MN, et al: Increased survival in pancreatic cancer with nab-paclitaxel plus gemcitabine. N Engl J Med 369: 1691-1703, 2013.

17. Ilic $M$ and Ilic I: Epidemiology of pancreatic cancer World J Gastroenterol 22: 9694-9705, 2016.

18. Yang JJ, Hu ZG, Shi WX, Deng T, He SQ and Yuan SG: Prognostic significance of neutrophil to lymphocyte ratio in pancreatic cancer: A meta-analysis. World J Gastroenterol 21: 2807-2815, 2015

19. Li H, Rokavec M and Hermeking H: Soluble IL6R represents a miR-34a target: Potential implications for the recently identified IL-6R/STAT3/miR-34a feed-back loop. Oncotarget 6: 14026-14032, 2015.

(i) (9) This work is licensed under a Creative Commons EY No No Attribution-NonCommercial-NoDerivatives 4.0 International (CC BY-NC-ND 4.0) License. 